Cysts were removed with spinal fixation. All the patients showed relapses and needed at least three surgical interventions.

Conclusion: Bone echinococcosis is rare and often misdiagnosed. Radiographic and CT images lack disease-specific characteristics whereas MRI images offers a greater chance of direct diagnosis. Treatment of spinal hydatid disease is entirely surgical with high risk of relapses.

Disclosure of Interests: None declared

DOI: 10.1136/annrheumdis-2021-eular.3986

\section{AB0785 TREATING IDIOPATHIC RECURRENT PERICARDITIS WITH INTERLEUKIN-1 INHIBITORS - A SINGLE CENTER EXPERIENCE}

P. Andel ${ }^{1,2,3}$, S. Brådland ${ }^{3}$, G. Haugeberg ${ }^{3,4} .{ }^{1}$ Ostfold Hospital Trust, Department of Cardiology, Sarpsborg, Norway; ${ }^{2}$ University of Oslo, Department of Clinical Medicine, Oslo, Norway; ${ }^{3}$ Hospital of Southern Norway, Department of

Rheumatology, Kristiansand, Norway; ${ }^{4}$ Norwegian University of Science and Technology, Department of Neuromedicine and Movement Science, Faculty of Medicine and Health Sciences, Trondheim, Norway

Background: Pericarditis is a common disease with significant morbidity (1). Idiopathic pericarditis, where an underlying cause cannot be identified, makes up for $80 \%$ of cases in the Western World (1). Up to $30 \%$ of these patients experience recurrence despite optimal treatment (2). Idiopathic recurrent pericarditis (IRP) is thought to represent an auto-inflammatory process rather than a reinfection (3). 2015 European Society of Cardiology (ESC) guidelines have outlined treatment of acute episodes and first recurrence with nonsteroidal anti-inflammatory drugs (NSAID), acetyl salicylic acid (ASS) and Colchicine as first line and Glucocorticoids (GC) as second line treatment (3). However GC treatment increases the risk of relapse, dependence and toxicity (2). Interleukin-1 (IL-1) inhibitors have been proposed as possible treatments in IRP $(3,4)$

Objectives: The aim of this case study is to outline our first experiences treating IRP with the IL-1 inhibitor anakinra in our Rheumatologic clinic.

Methods: All patients referred to our department in 2018/2019 with pericarditis were physically seen in our outpatient clinic. All patients were screened for malignancy, infection or rheumatologic disease as possible cause by clinical measures. Following ESC guideline, patients who suffered either the third recurrence under optimal treatment or significant side effects or dependency from GC were considered for anakinra treatment. Daily injection of anakinra $(100 \mathrm{mg})$ were given continuously over at least three months with gradual tapering over at least three months afterwards. Physical emergency department contacts, days hospitalized, colchicine- and GC use, the year prior to Anakinra treatment was recorded retrospectively. During follow up the same data was prospectively recorded.

Results: Over the course of two years 20 patients were referred to our clinic. All fulfilled ESC diagnostic criteria for pericarditis at index episode. In none of the patients could a rheumatologic, infectious or malignant cause be identified. 16 patients could be treated according to 2015 ESC guidelines with first or second line agents. Four patients were aligned to anakinra-treatment. Prior to referral, duration of symptoms was 5 - 120 months (mean 61 months). Further relevant patient- characteristics are outlined in Table 1.

After initiation of anakinra patients were afterwards regularly followed up in scheduled visits every 3 months.

Table 1. Characteristics of the four patients aligned to anakinra prior to anakinra-initiation.

\begin{tabular}{lccccc}
\hline Patient & $\begin{array}{c}\text { Number of } \\
\text { recurrences }\end{array}$ & $\begin{array}{c}\text { Number emergency } \\
\text { hospital contacts related } \\
\text { to IRP the year prior to } \\
\text { nakinra }\end{array}$ & $\begin{array}{c}\text { Days hospitalized GC dose } \\
\text { related to IRP } \\
\text { the year prior to } \\
\text { anakinra }\end{array}$ & $\begin{array}{c}\text { other } \\
\text { anakinra }\end{array}$ & \\
\hline I & 2 & 2 & 3 & $7.5 \mathrm{mg}$ steroidglau- \\
coma
\end{tabular}

Follow-up after start of anakinra was 6-15 months (mean 11.5 months). No patient was admitted to hospital or emergency department in that period. All four patients could taper and stop GC without recurrence. One patient experienced a mild relapse after discontinuing anakinra and was restarted on a low dose with complete remission. No patient had elevated CRP values at the end of follow-up and no patient experienced tamponade or clinical signs of constriction. No significant side effects were noted, no patient had to stop anakinra-treatment during follow up.
Conclusion: Implementation of anakinra treatment in cases of complicated IRP was both secure and successful in our rheumatologic outpatient department. In our small sample we could confirm findings from bigger trials regarding effectand side effect rates of anakinra treating IRP.

REFERENCES:

[1] Klein A et al. Cardiol Rev. 2020 Epub ahead of print. PMID: 32956167.

[2] Cremer P et al. J Am Coll Cardiol. 2016;68(21):2311-2328

[3] Adler Y et al European Heart Journal, 2015;36(42):2873-2885

[4] Imazio M et al Eur J Prev Cardiol. 2020;27(9):956-964

Disclosure of Interests: None declared

DOI: 10.1136/annrheumdis-2021-eular.4037

\section{AB0786 $\quad$ HUGHES-STOVIN SYNDROME: A PECULIAR AUTOIMMUNE ORIGIN OF PULMONARY ANEURYSMS}

P. Triggianese ${ }^{1}$, A. D'antonio ${ }^{1}$, B. Kroegler ${ }^{1}$, A. Ascoli Marchetti ${ }^{2}$, L. Belvivere ${ }^{2}$, D. Morosetti ${ }^{3}$, F. Sabuzi ${ }^{3}$, A. Ippoliti ${ }^{2}$, R. Argiro ${ }^{3}$, R. Perricone ${ }^{1} .{ }^{1}$ Rheumatology Allergology and Clinical Immunology, Department of "Medicina dei Sistemi", University of Rome Tor Vergata, Rome, Italy; ${ }^{2}$ Vascular Surgery Unit, Biomedicine and Prevention Department, University of Rome Tor Vergata, Rome, Italy; ${ }^{3}$ Diagnostic Imaging and Interventional Radiology, University Hospital of Rome Tor Vergata, Rome, Italy

Background: Hughes-Stovin Syndrome (HSS) is a rare potentially fatal vasculitis supposedly belonging to the spectrum of Behçet disease without ocular involvement. HSS tends to play by a temporal pattern, starting with thrombosis and followed by formation of pulmonary aneurysms. Since its mortality can reach $25 \%$ of cases, the early recognition and the appropriate therapy represent the major challenges.

Objectives: In this report, we aimed at describing a rare case of HSS successfully treated in accordance with a prompt combined management with endovascular approach and immunosuppressive treatments.

Methods: We described a Caucasian 33-year-old man presented to the Emergency Room of our Hospital with hemoptysis associated with dyspnea and chest pain, without fever and hypotension, with a 2-year history of painfu genital and oral ulcers. Blood test revealed anemia, elevated white cell count with increased C-reactive protein $(77 \mathrm{mg} / \mathrm{L})$ and D-dimer $(2740 \mathrm{ng} / \mathrm{mL})$. Doppler ultrasonography of legs showed thrombosis of the left femoral vein, while CT-angiography reported multiple aneurysms of the pulmonary arteries. An early and extensive work-up for infections and autoimmunity did not document relevant abnormalities. An appropriate genetic assessment revealed the HLA-B51 positivity. The patient immediately underwent endovascular embolization with coils and plugs of the largest pulmonary aneurysm, and inferio vena cava filter placement. Then, he started methylprednisolone $(1 \mathrm{mg} / \mathrm{kg} /$ day) IV for three days in association with LMWH (6000 IU/day). Prednisone $(1 \mathrm{mg} / \mathrm{kg} /$ day) in combination with cyclophosphamide (100 mg daily) were started orally, with tapering of steroids within a month. At the tight follow up (1 month and 3 months later), no genital and oral ulcers nor vascular thrombosis occurred, and acute phase reactants were in normal range. The 3-month-CT angiography showed a complete resolution of the aneurysms and no new changes.

Results: The peculiar diagnosis of HLAB51 positive HSS with multiple pulmonary aneurysms was made. The appropriate imaging followed by a prompt endovascular embolization of aneurysms with LMWH treatment were successfully performed. Interestingly, the oral combination therapy with corticosteroid and cyclophosphamide showed a rapid efficacy with a relevant safety profile. At the tight follow up, the young man improved significantly with clinical signs and pulmonary changes (Figure 1 next page).

Conclusion: This case is of interest because of the early recognition of such a rare disease that allowed an adequate combined radiological, vascular, and rheumatologic approach. In our case, LMWH resulted useful in preventing the pulmonary embolism, despite the use of anticoagulants is still debated in HSS Moreover, the endovascular embolization let a less invasive approach to surgery without the need for multiple surgical procedures. For the first time, we documented that oral cyclophosphamide showed an early efficacy as a first line therapy of a HLA-B51 HSS.

\section{REFERENCES:}

[1] Keskin M, Polat G, Ayrancı A, et al. Insidious Hughes Stovin Syndrome: Journey From Pulmonary Embolism to Pulmonary Arterial Aneurysm. Turk Thorac J. 2020;21:350-353.

[2] Valdés-Corona LF, Kimura-Hayama E, Méndez-Cano VH, et al. HughesStovin syndrome: an uncommon cause of pulmonary aneurysms. Rheumatology (Oxford). 2020;59:2183-2184. 


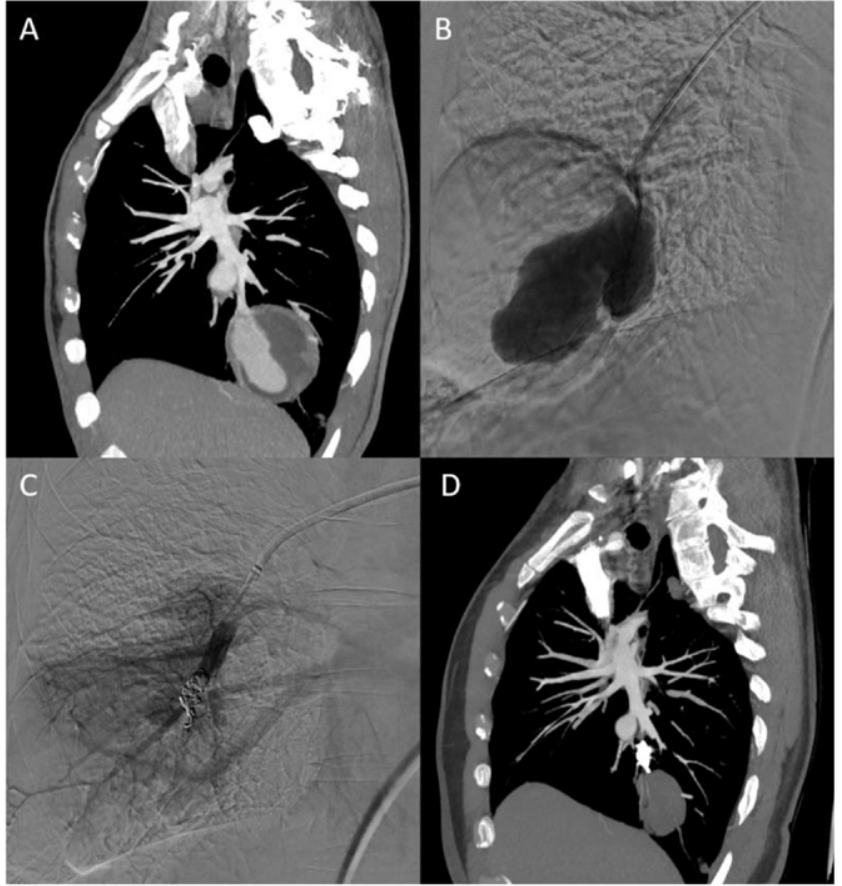

A. A large, partly-thrombosed aneurysm of the right pulmonary artery is shown, as well as a smaller aneurysm. B-C. Right pulmonary artery aneurysm in the lower lobe, before and after endovascukar embolization with coils and plug. D. Three-months CT scan after embolization.

Figure 1.

Disclosure of Interests: None declared

DOI: 10.1136/annrheumdis-2021-eular.4097

\section{Diagnostics and imaging procedures}

\section{AB0787 RECEIVER OPERATING CHARACTERISTIC ANALYSIS OF JOINT INFLAMMATION IN RELATION TO DISEASE ACTIVITY IN RHEUMATOID ARTHRITIS ASSESSED USING A NOVEL COMBINED THERMAL AND ULTRASOUND IMAGING}

Y. K. Tan ${ }^{1}$, C. Hong ${ }^{1}$, H. LI' , J. C. Allen $\mathrm{Jr}^{3}$, J. Thumboo ${ }^{1} .{ }^{1}$ Singapore General Hospital, Department of Rheumatology and Immunology, Singapore, Singapore; ${ }^{2}$ Singapore General Hospital, Health Services Research Unit, Singapore, Singapore; ${ }^{3}$ Duke-NUS Medical School, Centre for Quantitative Medicine, Singapore, Singapore

Background: A novel combined thermal and ultrasound (CTUS) imaging approach in rheumatoid arthritis (RA) was recently shown to be superior to either imaging modality alone in terms of correlation with the 28-joint disease activity score (DAS28). Objectives: To determine the performance of CTUS imaging in identifying RA patients with at least moderate disease activity (DAS28 > 3.2).

Methods: Bilateral hand (22 joints) thermal and ultrasound (US) imaging was performed. Thermal imaging provides the surface temperature readings at the joints with MAX, AVG and MIN derived per patient by summing the temperature differences with a control temperature, for the respective maximum (Tmax), average (Tavg) and minimum (Tmin) temperatures at each joint. US imaging assesses joint inflammation by summing up the power Doppler (PD) and grey-scale (GS) joint inflammation scores (graded 0-3 at each joint recess) at each joint to obtain the respective total PD and total GS scores per patient. CTUS imaging utilizes data from both thermal and US imaging to derive the MAX (PD), AVG (PD) and MIN (PD) by multiplying MAX, AVG and MIN by a factor of 2 when a patient's Total $\mathrm{PD}>$ median score, which otherwise remained the same as the MAX, AVG and MIN. The results of the imaging parameters were compared between patients with DAS28 $\leq 3.2$ and those with DAS28 $>3.2$. Sensitivity $(S n)$, specificity $(S p)$ and receiver operating characteristic $(\mathrm{ROC})$ curve analysis was performed to determine if the use of CTUS imaging can help identify patients with DAS28 $>3.2$.

Results: In this cross-sectional study, 814 joints from 37 RA patients $(75.7 \%$ female; $75.7 \%$ Chinese; baseline mean disease duration, 30.9 months; baseline mean DAS28, 4.43) were imaged. The mean (SD) values for the CTUS-but not single modality-imaging parameters (Table 1 ) were all significantly greater among patients with DAS28 $>3.2$ versus those with DAS28 $\leq 3.2$ (P-values were all $<0.01$ ). Based on cut-off levels of (a) MAX (PD) $\geq 94.5$, (b) MIN (PD) $\geq 42.3$ and (c) AVG (PD) $\geq 64.6$ in identifying patients with DAS28 $>3.2$, the respective area under the ROC curves (AUCs) $(95 \% \mathrm{Cls})$ were (a) $0.731(0.541,0.921)$ with $\mathrm{Sn}=58.1 \% ; \mathrm{Sp}=100.0 \%$; negative predictive value $(\mathrm{NPV})=31.6 \%$; positive predictive value $(\mathrm{PPV})=100.0 \%$; accuracy $=64.9 \%$, (b) $0.758(0.591,0.925)$ with $\mathrm{Sn}=61.3 \% ; \mathrm{Sp}=100.0 \% ; \mathrm{NPV}=33.3 \% ; \mathrm{PPV}=100.0 \%$; accuracy $=67.6 \%$ and (c) $0.763(0.596,0.931)$ with $\mathrm{Sn}=61.3 \% ; \mathrm{Sp}=100.0 \%$; NPV $=33.3 \%$; PPV $=100.0 \%$; accuracy $=67.6 \%$.

Conclusion: The severity of joint inflammation as detected by CTUS-but not single modality-imaging parameters were significantly greater among patients with DAS28 $>3.2$ versus those with DAS28 $\leq 3.2$. For the first time ever, by applying ROC analysis, this has helped to determine cut-off MAX (PD), MIN (PD) and AVG (PD) levels for identifying patients with DAS28 > 3.2; the usefulness of these cut-off levels will require further validation in independent RA cohorts.

Table 1. Comparison of imaging parameters between patient groups.

\begin{tabular}{|c|c|c|c|c|}
\hline \multirow{3}{*}{$\begin{array}{l}\text { Imaging } \\
\text { Parameter }\end{array}$} & \multicolumn{4}{|c|}{ DAS28 } \\
\hline & \multicolumn{2}{|c|}{ Mean $(95 \% \mathrm{Cl})$} & \multirow[t]{2}{*}{ Difference $(95 \% \mathrm{Cl})$} & \multirow[t]{2}{*}{ P-value } \\
\hline & DAS28 $\leq 3.2$ & DAS28 > 3.2 & & \\
\hline MAX (PD) & $75.25(58.8,91.7)$ & $119.5(101.12,137.87)$ & $-44.25(-70.49,-18.01)$ & $0.0022^{\star \star}$ \\
\hline MIN (PD) & $33.72(25.82,41.61)$ & $57.51(47.63,67.39)$ & $-23.79(-37.13,-10.45)$ & $0.0012^{\star \star}$ \\
\hline AVG (PD) & $50.72(39.45,61.98)$ & $85.39(71.89,98.88)$ & $-34.67(-53.27,-16.07)$ & $0.0008^{\star \star}$ \\
\hline MAX & $67.38(50.75,84.02)$ & $82.23(74.71,89.75)$ & $-14.85(-34.11,4.42)$ & 0.1268 \\
\hline MIN & $30.27(22.06,38.47)$ & $40.02(35.02,45.03)$ & $-9.75(-22.18,2.66)$ & 0.1198 \\
\hline AVG & $45.45(33.94,56.96)$ & $59.11(52.99,65.23)$ & $-13.66(-29.04,1.72)$ & 0.0801 \\
\hline Total PD & $2.83(-0.23,5.9)$ & $3.65(2.71,4.58)$ & $-0.82(-3.39,1.77)$ & 0.5269 \\
\hline Total GS & $6.67(1.31,12.02)$ & $6.58(4.12,9.04)$ & $0.09(-6.21,6.38)$ & 0.9780 \\
\hline
\end{tabular}

Statistically significant: ${ }^{\star \star} \mathrm{P}<0.01,{ }^{* \star \star} \mathrm{P}<0.001$.

Disclosure of Interests: None declared

DOI: 10.1136/annrheumdis-2021-eular.514

\section{$\mathrm{AB} 0788$}

MARKER OF CARTILAGE TISSUE LESION IMMUNOPATHOGENESIS OF RHEUMATOID ARTHRITIS

O. Rusanova ${ }^{1}$, A. Trofimenko ${ }^{2}$, N. Emelyanov ${ }^{2}$, O. Emelyanova ${ }^{1} .{ }^{1}$ Federal State Budgetary Institution «Research Institute of Clinical and Experimental Rheumatology named after A.B. Zborovsky», 1, Волгоград, Russian Federation; ${ }^{2}$ Federal State Budgetary Educational Institution of Higher Education "Volgoorad State Medical University» of the Ministry of Healthcare of the Russian Federation, 2, Волгоград, Russian Federation

Background: Collagen type 2 is the basic protein of cartilaginous tissue composing over $80 \%$ of its mass. Finding excessive antibodies to collagen type 2 and immune complexes is a diagnostic and prognostic criterion of immune lesion of articular cartilage.

Objectives: To study production of antibodies to collagen type 2 in patients with rheumatoid arthritis using immobilized magnetically controlled forms.

Methods: The antigen was represented by commercial formulation of collagen type 2 produced by Serva (Sweden). Antibodies to collagen type 2 were determined in the patients' blood serum by way of a technique of immunoenzyme assay (ELISA test) using immobilized magnetic sorbents. Magnetic sorbents were polyacrylamide granules from 10 to 100 micron in size containing magnetic material and collagen type 2 . We studied 30 apparently healthy donor individuals and 92 patients with a confirmed diagnosis of rheumatoid arthritis.

Results: A study of sera from rheumatoid arthritis patients revealed antibodies to collagen type 2 in 63 patients $(68.48 \%)$. The correlation coefficient between anti-collagen type 2 antibodies, and $\lg \mathrm{A} / \mathrm{IgM}$ amounted to 0.28 and 0.36 , correspondingly. At stage 1 and 2 of disease activity the level of antibodies was higher than in donors $(p<0.001)$. The highest level of antibodies to collagen type 2 was seen in patients with stage 3 of disease activity $(0.55 \pm 0.02)$. The amount of antibodies to collagen type 2 in patients with visceral manifestations of rheumatoid arthritis was no different from that in patients without any additional organ involvement, which is hardly surprising since collagen type 2 is mostly localized in articular cartilage and is practically absent from the connective tissue of other organs and systems.

Conclusion: Thus the presence of antibodies to collagen type 2 correlates with the disease activity and is an important marker of articular cartilage lesion in patients with rheumatoid arthritis.

Disclosure of Interests: None declared DOI: 10.1136/annrheumdis-2021-eular.828 